\title{
Transoral Thyroidectomy: Advantages and Disadvantages
}

\author{
Wan Wook Kim, M.D., Ph.D. \\ Department of Surgery, School of Medicine, Kyungpook National University, Daegu, Korea
}

\begin{abstract}
To date, many remote-access thyroid surgery techniques using endoscopic or robotic instruments have been developed. Transoral thyroidectomy has attracted great attention as a scarless thyroidectomy and is performed worldwide. Each surgical method has its advantages and disadvantages: selecting the optimal surgical method according to each patient's condition and preferences will possibly result in obtaining the best results and achieving patient satisfaction while minimizing complications after surgery.
\end{abstract}

Keywords: Remote access, Thyroidectomy, Transoral

\author{
Received July 30, 2020 \\ Revised August 25, 2020 \\ Accepted August 26, 2020 \\ Corresponding author \\ Wan Wook Kim \\ Department of Surgery, Kyungpook \\ National University Chilgok Hospital, \\ School of Medicine, Kyungpook \\ National University, 807 Hoguk-ro, \\ Buk-gu, Daegu 41404, Korea \\ Tel: +82-53-200-2705 \\ Fax: +82-53-200-2027 \\ E-mail: kww1324@naver.com \\ ORCID: \\ https://orcid.org/0000-0002-7363-5889
}

Copyright $(2020$ The Journal of Minimally Invasive Surgery. All rights reserved.
This is an Open Access article distributed under the terms of the Creative Commons Attribution Non-Commercial License (http://
creativecommons.org/licenses/by-nc/4.0/) which permits unrestricted non-commercial use, distribution, and reproduction in any medium, provided the original work is properly cited.
Techniques in thyroid surgeries using endoscopic and robotic methods include the transaxillary approach, the bilateral axillobreast approach (BABA), and the retroauricular approach. ${ }^{1-3}$ One of the advantages of such surgical methods is that they leave no visible scar on the neck, compared to the conventional open thyroidectomy. However, accessing the thyroid from a different site resulted in a longer incision on other sites, wider flap dissection range, and longer operation time. In the transaxillary and the retroauricular approaches, a $5 \sim 6 \mathrm{~cm}$, or more, incision scar can be seen in axilla or hairline behind the ear. These approaches have a major drawback; that is, operating on the contralateral thyroid is challenging because the incision is only made on one side. On the other hand, BABA has several advantages as follows: total thyroidectomy is easily performed using this technique as it provides access from both sides; scars are almost invisible because the incision in the areolar and axilla areas is approximately $1 \mathrm{~cm}$. However, it has a major disadvantage; that is, accessing the deep central neck area for lymph node dissection is challenging especially in the endoscopic method. ${ }^{4}$

The ideal remote-access thyroid surgery approach has to be technically easy to learn, reduce the operating time, and minimize the area of surgical dissection to reduce postoperative pain; in addition, both thyroidectomy and central lymph node dissection should be easily performed under a good surgical view; and, lastly, the scar should not be hidden but completely invisible. From the endoscopic and robotic thyroid surgeries that have been performed so far, the approach that meets all the above criteria is the transoral approach.

In recent years, many research papers have been published on the outcomes of the transoral approach. ${ }^{5-8}$ According to the results, when the technique was first introduced, a learning curve was observed; however, after that, the operating times between approaches were not significantly different. Moreover, the rates of adverse events were also acceptable, including intraoperative 
bleeding, length of stay, recurrent laryngeal nerve palsy, hypoparathyroidism, and infection. ${ }^{7}$ Transoral approach, with a lesser range of dissection, had postoperative pain similar to or less than other surgical methods. As a specific complication, mental nerve injury was reported in $4.3 \%$ of the patients. ${ }^{6}$ When the transoral approach was modified to the vestibular approach, the incidence of complications was greatly reduced. To reduce stretching injury or tearing of mental nerve, excessive movement of both lateral instruments should be minimized.

The advantages of transoral thyroidectomy are as follows: the external branch of the superior laryngeal nerve could be easily identified and preserved. ${ }^{9}$ Since the recurrent laryngeal nerve is first preserved near Berry's ligament and nerve is dissected downward, and then thyroid is pulled to the opposite side, the traction injury of the recurrent laryngeal nerve is minimized during thyroidectomy. Superior parathyroid gland and vasculature can be especially well preserved through capsular dissection in the craniocaudal direction; Transient hypocalcemia occurred in $5.6 \%$ of cases and permanent hypocalcemia was $0.1 \%$ in systemic review. Total thyroidectomy and central lymph node dissection are easily performed under ultimate craniocaudal view. This approach has a lesser surgical dissection range, achieving the highest satisfaction rate with the best cosmetic results as it is a scarless surgery. ${ }^{8}$ The disadvantages of transoral thyroidectomy are as follows: the modified radical neck dissection was not possible and superior pole dissection is challenging, particularly, when a goiter with a high superior pole or cancer is located at the upper pole end, especially with an extrathyroidal extension. Access to the superior pole when bleeding is difficult; thus, surgeons must be careful in dissecting the superior pole. Moreover, if the patient's mouth is too small, the movements of the instruments could be restricted, and if the mandible is large and protruding, accessing the surgical space may be difficult.

The indications of the transoral thyroidectomy were a well differentiated thyroid cancer less than $2 \mathrm{~cm}$ in diameter, benign mass or follicular neoplasm less than $4 \mathrm{~cm}$. Thyroiditis, Graves' disease and central lymph node metastasis were not contraindications of transoral thyroidectomy. Contraindications were poorly differentiated or undifferentiated cancer, lateral lymph node metastasis and invasion in surrounding important structures (trachea, recurrent laryngeal nerve, and esophagus). Relative contraindications were too small size of the mouth (maximum size $3.0 \sim 3.5 \mathrm{~cm}$ or less), the severe protruding chin, and the cancer located at upper pole and fixed with extrathyroidal extension.

As each surgical method has its benefits and limitations, the surgical method should be determined based on the patient's condition and preferences to achieve the best surgical results and patient satisfaction.

\section{ORCID}

Wan Wook Kim, https://orcid.org/0000-0002-7363-5889

\section{CONFLICT OF INTEREST}

None.

\section{FUNDING}

None.

\section{ACKNOWLEDGMENTS}

None.

\section{REFERENCES}

1) Choe JH, Kim SW, Chung KW, et al. Endoscopic thyroidectomy using a new bilateral axillo-breast approach. World J Surg 2007;31:601-606.

2) Kang SW, Lee SC, Lee SH, et al. Robotic thyroid surgery using a gasless, transaxillary approach and the da Vinci S system: the operative outcomes of 338 consecutive patients. Surgery 2009;146:10481055.

3) Byeon HK, Kim da H, Chang JW, et al. Comprehensive application of robotic retroauricular thyroidectomy: The evolution of robotic thyroidectomy. Laryngoscope 2016;126:1952-1957.

4) Shan L, Liu J. Meta-analysis Comparison of Bilateral Axillo-Breast Approach Robotic Thyroidectomy and Conventional Thyroidectomy. Surg Innov 2019;26:112-123.

5) Anuwong A, Ketwong K, Jitpratoom P, Sasanakietkul T, Duh QY. Safety and Outcomes of the Transoral Endoscopic Thyroidectomy Vestibular Approach. JAMA Surg 2018;153:21-27.

6) Shan L, Liu J. A Systemic Review of Transoral Thyroidectomy. Surg Laparosc Endosc Percutan Tech 2018;28:135-138.

7) Chen S, Zhao M, Qiu J. Transoral vestibule approach for thyroid disease: a systematic review. Eur Arch Otorhinolaryngol 2019;276:297304.

8) Kim WW, Lee J, Jung JH, Park HY, Tufano RP, Kim HY. A comparison study of the transoral and bilateral axillo-breast approaches in robotic thyroidectomy. J Surg Oncol 2018;118:381-387.

9) Ji YB, Jeong JH, Wu CW, Chiang FY, Tae K. Neural Monitoring of the External Branch of the Superior Laryngeal Nerve During Transoral Thyroidectomy. Laryngoscope 2020 Jul 17 [Epub]. https://doi. org/10.1002/lary.28883. 